From the Division of Cancer Medicine: and the Department of Breast Medical Oncology, The University of Texas M. D. Anderson Cancer Center, Houston, TX

Submitted April 9, 2008; accepted December 10, 2008; published online ahead of print at www.jco.org on March 23, 2009

Supported in part by Grant No. 1K07CA109064-05 from the National Institutes of Health (S.H.G.)

Authors' disclosures of potential conflicts of interest and author contributions are found at the end of this article.

Corresponding author: Sharon $\mathrm{H}$ Giordano, MD, Dept of Breast Medical Oncology, Unit 1354, The University of Texas M. D. Anderson Cancer Center, 1515 Holcombe Blvd, Houston, TX 77030-4009, e-mail: sgiordan@ mdanderson.org

(c) 2009 by American Society of Clinical Oncology

0732-183X/09/2713-2170/\$20.00

DOI: $10.1200 / J C O .2008 .17 .5935$

\title{
Impact of Diabetes Mellitus on Complications and Outcomes of Adjuvant Chemotherapy in Older Patients With Breast Cancer
}

Tomasz P. Srokowski, Shenying Fang, Gabriel N. Hortobagyi, and Sharon H. Giordano

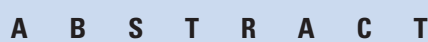

\section{Purpose}

To evaluate whether diabetes affects patterns of adjuvant chemotherapy use, toxic effects of chemotherapy, and breast cancer outcomes.

\section{Patients and Methods}

By using the Surveillance, Epidemiology, and End Results-Medicare database, we identified patients aged 66 years or older who had stages I through III breast cancer that was diagnosed between 1992 and 2002. Multivariable regression analyses were performed to determine the effect of diabetes on use of chemotherapy, toxicities, and outcomes. The risks of all-cause mortality and breast cancerspecific (BCS) mortality were estimated with the Kaplan-Meier method.

\section{Results}

Our cohort had 70,781 men and women, of whom 14,414 (20.36\%) had diabetes. Among people who received chemotherapy $(n=11,826), 21.0 \%$ were diabetics. In this group, diabetics had lower odds of receiving anthracyclines (odds ratio [OR], $0.78 ; 95 \% \mathrm{Cl}, 0.71$ to 0.87 ) and taxanes (OR, 0.86; 95\% Cl, 0.75 to 0.99). Diabetes was associated with increased odds of being hospitalized for any chemotherapy toxicity $(\mathrm{OR}, 1.38 ; 95 \% \mathrm{Cl}, 1.23$ to 1.56$)$, for infection or fever (OR, 1.43; 95\% Cl, 1.2 to 1.7), for neutropenia (OR, 1.22; 95\% Cl, 1.03 to 1.45), for anemia (OR, $1.24 ; 95 \% \mathrm{Cl}, 1.05$ to 1.47 ), and for any cause (OR, 1.32; $95 \% \mathrm{Cl}, 1.19$ to 1.46). Patients with diabetes had higher all-cause mortality (hazard ratio [HR], 1.35; 95\% $\mathrm{Cl}, 1.31$ to 1.39). There was a significant interaction between diabetes and chemotherapy use for BCS mortality. Diabetic and nondiabetic patients who did not receive chemotherapy had similar BCS mortality, but diabetic patients who did receive chemotherapy had higher BCS mortality than nondiabetic patients (OR, $1.20 ; 95 \% \mathrm{Cl}, 1.07$ to 1.35$)$.

\section{Conclusion}

In this observational, hypothesis-generating study, patients who have breast cancer and diabetes are at increased risk of chemotherapy-related toxicities compared with nondiabetic patients who are receiving chemotherapy and have higher all-cause mortality.

\section{J Clin Oncol 27:2170-2176. (c) 2009 by American Society of Clinical Oncology}

\section{INTRODUCTION}

Diabetes mellitus and breast cancer are common diseases and major health care problems in developed countries. The relationship between diabetes and breast cancer is complex and not well understood. Diabetes is associated with multiple factors that can potentially influence breast cancer, such as comorbidities, obesity, fitness, and metabolic syndrome. Diabetes may affect breast cancer directly by contributing to breast cancer risk on a molecular level, by mechanisms such as altered endogenous sex-hormone regulation and activation of the insulin-like growth factor and insulin-signaling pathways. ${ }^{1}$ Diabetes also may have indirect effects on breast cancer because of associated end organ damage that may influence screening and treatment choices, affect treatment toxicities, and lead to worse outcomes.

Published data on the effect of diabetes on treatment-related toxicities and outcomes in breast cancer are limited. Diabetes has been associated with an increased risk of complications after breast cancer surgery, ${ }^{2,3}$ but no studies to date have reported on complications of breast cancer chemotherapy in patients with diabetes. The two studies ${ }^{4,5}$ that examined the effect of diabetes on breast cancer outcomes yielded conflicting results. Those studies varied substantially in population, size, and degree of adjustment for confounding factors. 
Given the lack of data about use and complications of chemotherapy in patients with breast cancer and diabetes, and given conflicting data about breast cancer outcomes in patients with diabetes, we conducted this study to evaluate whether diabetes affects patterns of adjuvant chemotherapy use, toxic effects of chemotherapy, and outcomes in patients with breast cancer.

\section{PATIENTS AND METHODS}

\section{Data Source}

We used the Surveillance, Epidemiology, and End Results (SEER)Medicare linked database for this study. The SEER program, supported by the US National Cancer Institute (NCI), collects data from tumor registries; during the years included in this study, the database covered $14 \%$ to $25 \%$ of the US population. ${ }^{6}$ The Medicare program is administered by the Centers for Medicare and Medicaid Services and covers $97 \%$ of the US population aged 65 years or older. ${ }^{7}$ SEER participants are matched with their Medicare records under an agreement between the NCI and Centers for Medicare and Medicaid Services. Of SEER participants who were diagnosed with cancer at age 65 years or older, $94 \%$ are matched with their Medicare enrollment records. ${ }^{7}$

In this study, breast cancer patients diagnosed between 1992 and 2002 were linked with their Medicare claims, which were available through 2004. Patient demographics, tumor characteristics, and treatment information were extracted from the SEER-Medicare Patient Entitlement and Diagnosis Summary File.

\section{Study Population}

In the SEER-Medicare database, 212,477 men and women were diagnosed with breast cancer from January 1992 to December 2002. This study included patients aged 66 years or older who had a diagnosis of stages I through III breast cancer (according to American Joint Committee on Cancer staging system, modified third edition). We included only patients who were treated with definitive surgical therapy. Patients who had a previous cancer were excluded. Patients were required to have Medicare Part A and Part B and to not be members of a Health Maintenance Organization (HMO) for 1 year prior and after their breast cancer diagnoses, because Medicare claims are not complete for HMO members. All patients with noncarcinoma tumor histology represented by codes 8800 to 9758 also were excluded.

The numbers of patients excluded from our final study population were as follows: 6,036 patients with a previous cancer diagnosis; 731 with an unknown date of diagnosis; 66,174 who were younger than 66 years old at the time of diagnosis; 33,003 with tumor stages other than I through III; 2,433 who did not receive definitive surgery; 33,162 patients who were not covered by Medicare or who were not members of an HMO for the year before or after diagnosis, and 157 who had noncarcinoma histology.

\section{Data Extraction and Definitions}

Diabetic status was determined on the basis of the International Classification of Disease (ICD)-9-clinical modification diagnosis code of $250 \times$ in Medicare inpatient, outpatient, physician, or Durable Medical Equipment (DME) files within 12 months before breast cancer diagnosis. Patients were classified as having diabetes if at least one diagnosis code of diabetes was identified in any claim file. This method was previously shown to have $72 \%$ sensitivity and $96 \%$ specificity in identifying patients with diabetes in Medicare claims data. ${ }^{8}$

To identify patients who received adjuvant chemotherapy and to determine the chemotherapy regimens used, we looked for chemotherapy-related codes in Medicare claims within 6 months of breast cancer diagnosis. The following codes were used for defining chemotherapy regimens: ICD-9-CM procedure code 9925 for a hospital inpatient or outpatient facility claim of chemotherapy; the Common Procedure Terminology codes J8510, J8520, J8521, J8530 through J8999, and J9000 through J9999, excluding J9202 (goserelin), J9209 (mesna), J9212 through J9214 (interferon), J9217-J9218 (leuprolide acetate), in physician, outpatient, or DME files; revenue center codes 0331, 0332, and 0335 for an outpatient claim of chemotherapy; and the ICD-9-CM V codes V58.1, V66.2, or V67.2 in inpatient, outpatient, physician, or DME files. We classified chemotherapy as an anthracycline-based regimen if J codes for doxorubicin (ie, J9000, J9010, J9001, J9150, J9151, J9178, J9180) or mitoxantrone (ie, J9293) were present and as a taxane-based regimen if J codes for paclitaxel (ie, J9265) or docetaxel (ie, J9170) were identified.

Toxicity related to chemotherapy was defined in three ways. First, we evaluated the rates of hospitalization from any cause within 6 months of starting chemotherapy. We performed a secondary analysis to evaluate hospitalizations within 6 months of chemotherapy initiation with any of the following discharge diagnoses, which indicated chemotherapy toxicity: infection (001.0 to 139.8), neutropenia (288.0), dehydration (276.5), fever (780.6), anemia (284.0 to 285.9), thrombocytopenia (287.4), delirium (780.X), or unspecified toxicity (E9331). We used a previously published algorithm to identify chemotherapy toxicities, which included relevant discharge diagnosis codes in any position. ${ }^{9}$ Finally, we evaluated the risk of hospitalization for the individual toxicities of infection, fever, neutropenia, and anemia.

By using Medicare inpatient, outpatient, and physician claims data, we searched for ICD-9-CM diagnosis and procedure codes that indicated the presence of comorbid conditions from 12 to 3 months before diagnosis of the breast cancer. A comorbidity score that excluded the contribution of diabetes was calculated for each patient by using Klabunde's adaptation of the Charlson comorbidity index from the SAS macro provided by the NCI. ${ }^{10-12}$

Adjuvant radiotherapy use was identified through either SEER or SEERMedicare claims data. To be considered adjuvant radiotherapy, the radiotherapy claim had to begin within 6 months of the breast cancer diagnosis. For the census tract variables of education and poverty level, quartiles were calculated in increasing order.

Data from the 2000 census were supplemented with 1990 data if 2000 data were missing. Patients were followed up from diagnosis date until December 2004, loss of Medicare coverage, enrollment in HMO, or death.

\section{Statistical Analyses}

The distributions of patients' demographic and tumor characteristics were compared between patients with and without diabetes, and differences were assessed with the $\chi^{2}$ test.

To determine patterns of adjuvant chemotherapy use, multivariable logistic regression models were built for the odds of receiving chemotherapy and the odds of receiving treatment with anthracyclines and taxanes (among patients who received chemotherapy). Models were adjusted for sex, age at diagnosis, ethnicity, marital status, education level, poverty level, year of diagnosis, SEER region, tumor grade, estrogen receptor (ER) status, number of positive lymph nodes, Charlson comorbidity index, type of surgery, and diabetes.

Among all patients who received chemotherapy, hospitalization and hospitalization for toxicities (within 6 months of initiating chemotherapy) were also treated as outcome variables and were analyzed using logistic regression that was adjusted for the above predictor variables (with the addition of chemotherapy regimen).

The probabilities of all-cause mortality and breast cancer-specific (BCS) mortality were estimated with the Kaplan-Meier method. Survival probabilities among all patients and patients who did and did not receive adjuvant chemotherapy were plotted stratified by diabetes status, and a test of equality over strata was performed by using the log-rank test. Proportional hazard ratios (HRs) for death were estimated when adjusting for sex, patient age, ethnicity, marital status, education level, poverty level, year of diagnosis, SEER region, tumor grade, ER status, number of involved lymph nodes, Charlson comorbidity index, type of surgery, use of chemotherapy, use of radiation, and diabetes.

All computer programming and statistical analyses were performed with the SAS system (Cary, NC).

\section{RESULTS}

\section{Baseline Characteristics}

The final cohort included 70,781 elderly patients with breast cancer, of whom 14,414 (20.4\%) had diabetes. Patient demographics 
Srokowski et al

\begin{tabular}{|c|c|c|c|c|}
\hline \multirow[b]{2}{*}{ Characteristic } & \multicolumn{3}{|c|}{ Patients } & \multirow[b]{2}{*}{$P$} \\
\hline & No. Overall & $\%$ Nondiabetic $(n=56,367)$ & $\%$ Diabetic $(n=14,414)$ & \\
\hline \multicolumn{5}{|l|}{ Sex } \\
\hline Female & 70,254 & 99.29 & 99.13 & .0549 \\
\hline Male & 527 & 0.71 & 0.87 & \\
\hline \multicolumn{5}{|l|}{ Age, years } \\
\hline $66-70$ & 17,448 & 24.98 & 23.35 & $<.0001$ \\
\hline $71-75$ & 18,985 & 26.67 & 27.43 & \\
\hline $76-80$ & 16,573 & 22.91 & 25.37 & \\
\hline$>80$ & 17,775 & 25.44 & 23.85 & \\
\hline \multicolumn{5}{|l|}{ Ethnicity } \\
\hline Black & 4071 & 4.53 & 10.52 & $<.0001$ \\
\hline Other & 4613 & 5.87 & 9.05 & \\
\hline White & 62,097 & 89.6 & 80.44 & \\
\hline \multicolumn{5}{|l|}{ Marital status } \\
\hline Married & 29,366 & 42.57 & 37.25 & $<.0001$ \\
\hline Not married & 39,011 & 54.08 & 59.15 & \\
\hline Unknown & 2404 & 3.34 & 3.6 & \\
\hline \multicolumn{5}{|c|}{ Census tract education } \\
\hline Highest quartile & 17,571 & 26.65 & 17.71 & $<.0001$ \\
\hline High quartile & 17,565 & 25.5 & 22.12 & \\
\hline Low quartile & 17,594 & 24.38 & 26.74 & \\
\hline Lowest quartile & 17,571 & 22.79 & 32.78 & \\
\hline Unknown & 480 & 0.68 & 0.65 & \\
\hline \multicolumn{5}{|l|}{ Census tract poverty } \\
\hline Lowest quartile & 17,635 & 25.68 & 21.94 & $<.0001$ \\
\hline Low quartile & 17,518 & 25.32 & 22.53 & \\
\hline High quartile & 17,570 & 24.99 & 24.18 & \\
\hline Highest quartile & 17,578 & 23.33 & 30.71 & \\
\hline Unknown & 480 & 0.68 & 0.65 & \\
\hline \multicolumn{5}{|l|}{ Year of diagnosis } \\
\hline 1992 & 5559 & 8.14 & 6.74 & $<.0001$ \\
\hline 1993 & 5256 & 7.72 & 6.27 & \\
\hline 1994 & 5098 & 7.35 & 6.64 & \\
\hline 1995 & 5138 & 7.5 & 6.33 & \\
\hline 1996 & 4960 & 7.16 & 6.42 & \\
\hline 1997 & 5010 & 7.18 & 6.67 & \\
\hline 1998 & 4971 & 7.05 & 6.91 & \\
\hline 1999 & 5064 & 7.05 & 7.56 & \\
\hline 2000 & 9853 & 13.63 & 15.06 & \\
\hline 2001 & 10,036 & 13.85 & 15.48 & \\
\hline 2002 & 9836 & 13.38 & 15.92 & \\
\hline \multicolumn{5}{|l|}{ Tumor stage } \\
\hline । & 40,624 & 57.57 & 52.78 & $<.0001$ \\
\hline$\|$ & 26,818 & 36.5 & 40.73 & \\
\hline III & 4335 & 5.93 & 6.49 & \\
\hline \multicolumn{5}{|l|}{ Tumor grade* } \\
\hline 1 & 13,377 & 19.51 & 16.52 & $<.0001$ \\
\hline 2 & 27,411 & 38.73 & 38.73 & \\
\hline 3 & 19,261 & 26.65 & 29.42 & \\
\hline Unknown & 10,732 & 15.12 & 15.34 & \\
\hline \multicolumn{5}{|l|}{ ER status } \\
\hline Negative & 8948 & 12.57 & 12.92 & .0006 \\
\hline Positive & 48,911 & 69.42 & 67.84 & \\
\hline Unknown & 12,922 & 18.01 & 19.23 & \\
\hline \multicolumn{5}{|l|}{ Tumor size, cm } \\
\hline $0-2.0$ & 47,265 & 67.78 & 62.86 & $<.0001$ \\
\hline $2.1-5.0$ & 19,333 & 26.41 & 30.85 & \\
\hline$>5.0$ & 3463 & 4.79 & 5.28 & \\
\hline Unknown & 720 & 1.02 & 1.01 & \\
\hline & & (continued on following page) & & \\
\hline
\end{tabular}




\begin{tabular}{|c|c|c|c|c|}
\hline \multirow[b]{2}{*}{ Characteristic } & \multicolumn{3}{|c|}{ Table 1. Patient, Disease, and Treatment Characteristics (continued) } & \multirow[b]{2}{*}{$P$} \\
\hline & No. Overall & $\%$ Nondiabetic $(n=56,367)$ & $\%$ Diabetic $(n=14,414)$ & \\
\hline \multicolumn{5}{|l|}{ Lymph node status } \\
\hline Negative & 39,242 & 56.2 & 52.46 & $<.0001$ \\
\hline Positive & 16,036 & 22.12 & 24.75 & \\
\hline Unknown & 15,503 & 21.68 & 22.79 & \\
\hline \multicolumn{5}{|l|}{ No. of positive nodes } \\
\hline 0 & 39,242 & 56.2 & 52.46 & $<.0001$ \\
\hline $1-3$ & 10,537 & 14.63 & 15.91 & \\
\hline $4-9$ & 3669 & 5.05 & 5.7 & \\
\hline$\geq 10$ & 1830 & 2.44 & 3.14 & \\
\hline Unknown & 15,503 & 21.68 & 22.79 & \\
\hline \multicolumn{5}{|l|}{ Comorbidity index } \\
\hline 0 & 57,718 & 82.67 & 70.22 & $<.0001$ \\
\hline 1 & 10,280 & 13.36 & 19.06 & \\
\hline $2+$ & 3783 & 3.97 & 10.72 & \\
\hline \multicolumn{5}{|c|}{ Adjuvant chemotherapy } \\
\hline No & 58,955 & 83.43 & 82.77 & .0581 \\
\hline Yes & 11,826 & 16.57 & 17.23 & \\
\hline \multicolumn{5}{|l|}{ Adjuvant radiotherapy } \\
\hline No & 36,972 & 51.83 & 53.82 & $<.0001$ \\
\hline Yes & 34,199 & 48.17 & 46.18 & \\
\hline \multicolumn{5}{|l|}{ Surgery } \\
\hline Breast-conserving & 35,207 & 50.56 & 46.55 & $<.0001$ \\
\hline Mastectomy & 35,574 & 49.44 & 53.45 & \\
\hline
\end{tabular}

are listed in Table 1. In multivariable analysis, diabetes was associated with small but significantly reduced odds of receiving chemotherapy (OR, $0.91 ; 95 \%$ CI, 0.85 to 0.96 ).

\section{Chemotherapy Regimens and Toxic Effects}

A total of 11,826 patients (16.7\% of the entire cohort) who received adjuvant chemotherapy were included in the analyses of chemotherapy use and toxicity. Among this cohort, 2,484 (21.0\%) were diabetics, and 9,342 (79.0\%) were not diabetics. In multivariable analysis of patients who received chemotherapy, diabetes was independently associated with reduced odds of receiving anthracyclines (OR, $0.78 ; 95 \% \mathrm{CI}, 0.71$ to 0.87 ) or taxanes (OR, 0.86 ; $95 \% \mathrm{CI}, 0.75$ to 0.99 ).

We next evaluated the association between diabetes and chemotherapy toxicity. Overall, 3,201 patients $(27.1 \%)$ who received adju- vant chemotherapy were hospitalized for any cause. $32.7 \%$ of patients with diabetes were hospitalized (Table 2). In the multivariable analysis, diabetes was associated with increased odds of hospitalization for any cause; hospitalization for chemotherapy toxicity; and hospitalization for infection or fever, neutropenia, or anemia (Table 3).

\section{Outcomes}

Breast cancer patients with diabetes were more likely to die of any cause than were nondiabetic breast cancer patients (Fig 1). In the multivariable analysis, there was a significant association between diabetes and all-cause mortality (HR 1.35; 95\% confidence interval 1.31-1.39).

Patients with diabetes were also more likely to as a result of breast cancer than were nondiabetic patients, although the absolute differences were small. Survival curves are shown in Figure 2. In

\begin{tabular}{|c|c|c|c|c|c|c|}
\hline \multirow{3}{*}{$\begin{array}{l}\text { Reason for } \\
\text { Hospitalization }\end{array}$} & \multicolumn{5}{|c|}{ Patients } & \multirow[b]{3}{*}{$P$} \\
\hline & \multirow[b]{2}{*}{ No. Overall } & \multicolumn{2}{|c|}{$\begin{array}{l}\text { Without Diabetes } \\
\qquad(\mathrm{n}=9,342)\end{array}$} & \multicolumn{2}{|c|}{$\begin{array}{l}\text { With Diabetes } \\
(\mathrm{n}=2,484)\end{array}$} & \\
\hline & & No. & $\%$ & No. & $\%$ & \\
\hline Any cause & 3,201 & 2,390 & 25.6 & 811 & 32.6 & $<.0001$ \\
\hline Neutropenia & 833 & 634 & 6.8 & 199 & 8.01 & .0340 \\
\hline Anemia & 858 & 624 & 6.7 & 234 & 9.4 & $<.0001$ \\
\hline
\end{tabular}


Table 3. Factors Associated With Increased Odds of Hospitalization for Any Cause and for Toxicities of Chemotherapy

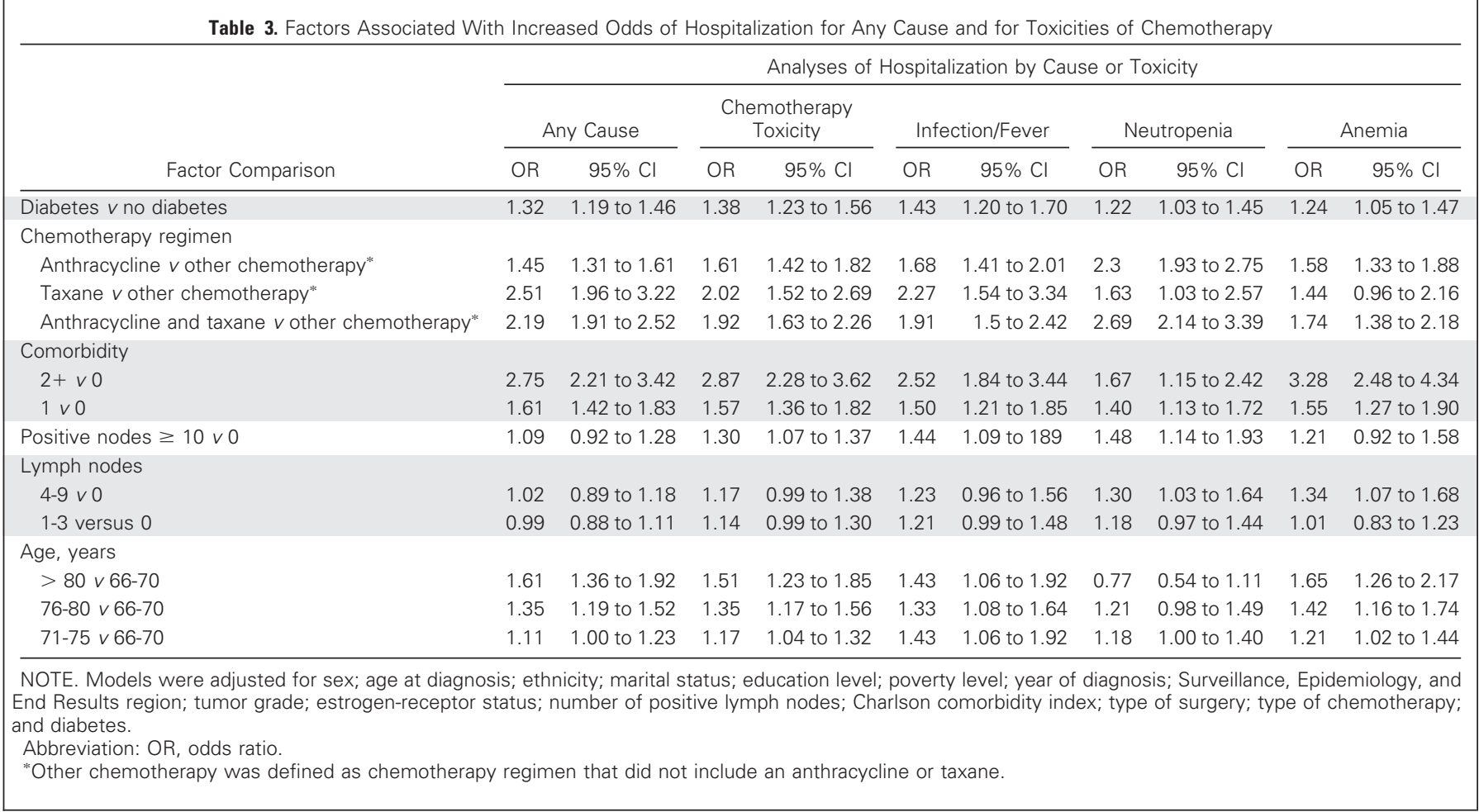

the multivariable analysis, we found that there was a significant interaction between diabetes and chemotherapy use for BCS mortality. When we stratified patients by chemotherapy use, the BCS mortality of diabetic patients who did not receive chemotherapy was similar to that of nondiabetic patients who did not receive chemotherapy $(\mathrm{HR}$, 0.95, 95\% CI, 0.88 to 1.03 ); however, diabetics who did receive chemotherapy had higher BCS mortality than nondiabetic patients who received chemotherapy ( $\mathrm{HR}, 1.20,95 \% \mathrm{CI}, 1.07$ to 1.35$)$.

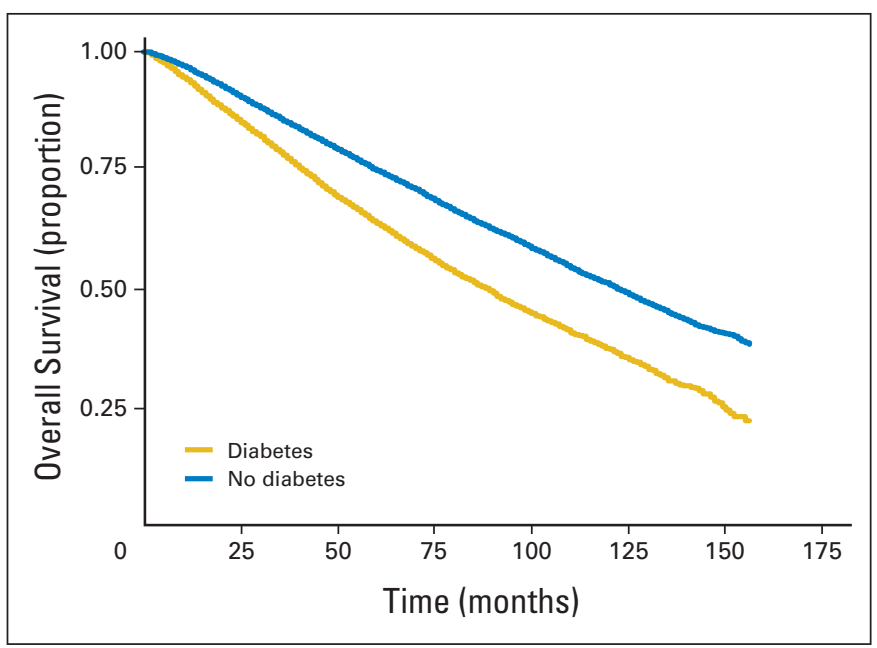

Fig 1. Unadjusted all-cause mortality curve of patients with and without diabetes and breast cancer. Five- and 10-year all-cause survival probabilities: 0.64 (95\% Cl, 0.63 to 0.65$)$ and $0.37(95 \% \mathrm{Cl}, 0.36$ to 0.39$)$, respectively, for patients with diabetes, and $0.75(95 \% \mathrm{Cl}, 0.75$ to 0.75$)$ and $0.51(95 \% \mathrm{Cl}, 0.50$ to 0.52$)$, respectively, for patients without diabetes $(P<.0001$ for each).

\section{DISCUSSION}

Diabetics constitute a large proportion of patients with breast cancer. In this cohort, $20.3 \%$ of patients had diabetes. This is similar to the prevalence of diabetes in the general population that was reported by the Centers for Disease Control and Prevention in 2005: 20.9\% among patients aged 60 years or older. ${ }^{13}$ In this study, patients with diabetes were more likely to have more advanced disease (ie, higher stage and grade) than nondiabetic patients. This may, in part, be explained by socioeconomic factors: patients with diabetes and breast cancer were also more likely to be less educated, to be from poorer neighborhoods, and to have more comorbidities. It is also possible that diabetic patients, because of the focus of their physicians on chronic diseases that frequently accompany the diabetes, receive less-frequent screening. Finally, as it has been recently recognized, diabetes may also affect severity of breast cancer directly by altering endogenous sex-hormone regulation and activation of the IGF and insulin-signaling pathways. ${ }^{1}$ In contrast, Guastamacchia et $\mathrm{al}^{14}$ found no association between diabetes and tumor grade, tumor size, or lymph node status, but that study included only a small number $(n=77)$ of patients with diabetes.

When we examined whether diabetic patients were more likely to experience toxicities of chemotherapy than were nondiabetic patients, we found that this was, in fact, the case. Diabetic patients were more likely to be hospitalized for any cause; for toxicity in general; and for infection or fever, neutropenia, and anemia. Meyerhardt et $\mathrm{al}^{15}$ did not find an increased incidence of treatment-related adverse events in diabetic versus nondiabetic patients who received adjuvant chemotherapy for colon cancer (only diarrhea was more common in diabetics). This difference may be due to the use of less-toxic chemotherapy regimens and to a younger study 


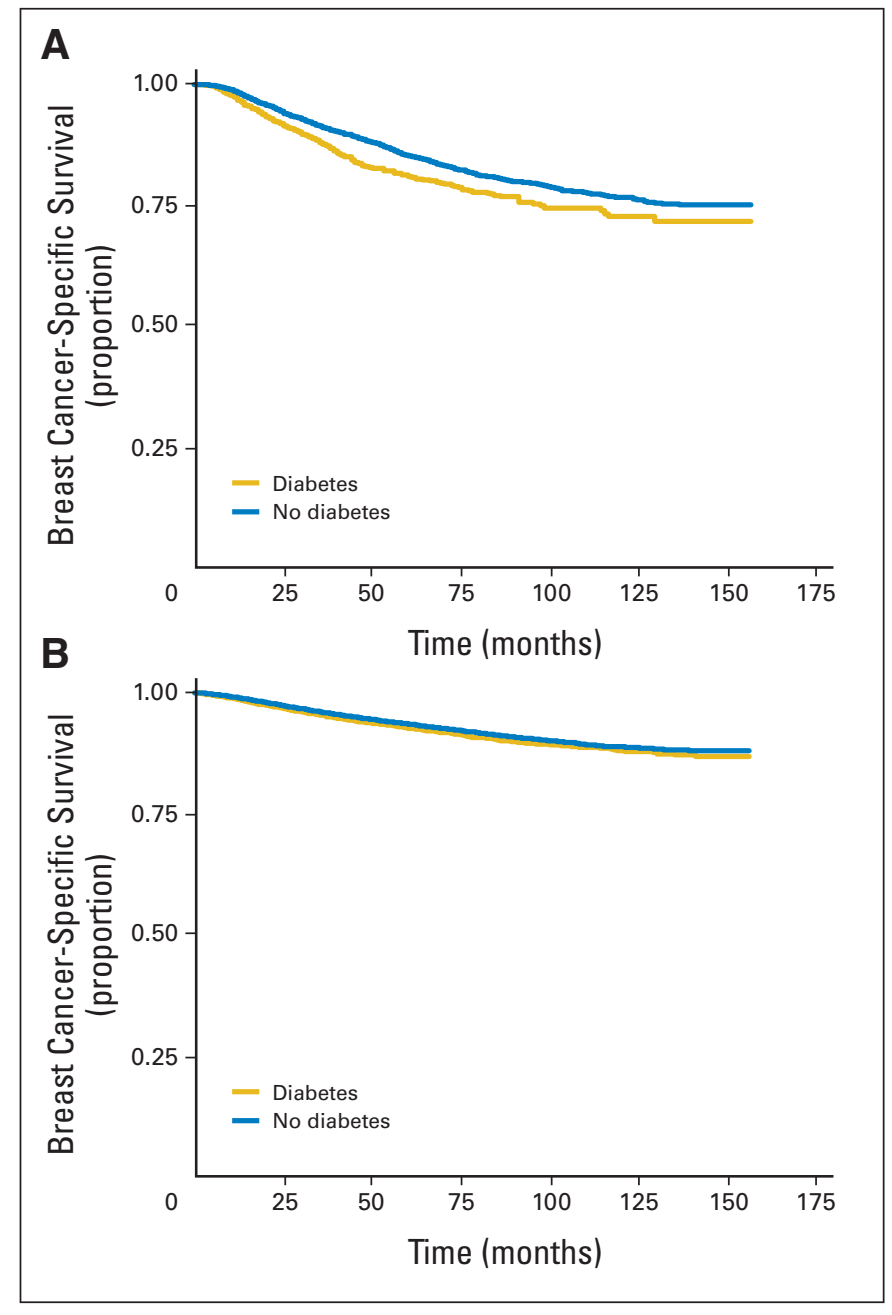

Fig 2. Unadjusted breast cancer-specific (BCS) mortality curve of patients with and without diabetes and breast cancer who $(A)$ received chemotherapy and who (B) did not receive chemotherapy.

population in the study by Meyerhardt et al. ${ }^{15}$ It is also possible that, because we relied on hospitalization records to assess toxicities, at least some difference in complication rates observed in this study may be caused by physicians who have a different threshold for hospitalization between diabetic and nondiabetic patients (ie, presumably patients who have diabetes were more likely to be admitted).

In this study, receipt of anthracycline-or taxane-containing chemotherapy was among the factors that affected the risk of hospitalization and developing toxicities (compared with other chemotherapy regimens) with magnitude of effect exceeding the influence of diabetes. It is important to point out that patients with diabetes were somewhat less likely to receive chemotherapy overall and also less likely to receive anthracycline- and taxane-based chemotherapy yet still experienced significantly higher rates of toxicities.

We found that older patients with breast cancer and diabetes are markedly more likely to die as a result of any cause than nondiabetic patients with breast cancer. This is consistent with the study of Yancik et al, ${ }^{5}$ who found that diabetes was correlated with an increased risk of death in breast cancer patients (risk ratio, 1.76; 95\% CI, 1.23 to 2.52). In contrast, Fleming et $\mathrm{al}^{4}$ did not find diabetes to be a significant risk factor for death in breast cancer patients; however, that study examined only 1-year mortality. When we examined BCS mortality, diabetic patients who received adjuvant chemotherapy had higher BCS mortality than nondiabetic patients who received adjuvant chemotherapy, whereas diabetic patients who did not receive chemotherapy had similar BCS mortality to nondiabetic patients who did not receive chemotherapy.

There are several potential explanations of why diabetes affected all-cause mortality more than BCS mortality. First, diabetes and its complications may account for the difference in all-cause mortality among diabetic and nondiabetic patients with breast cancer; diabetes is a well-known risk factor for many potentially fatal conditions, including cardiovascular disease. This explanation is supported by the study of Satariano and Ragland, ${ }^{16}$ who found an association between comorbidities and risk of death in patients with breast cancer. In addition, in the study by Yancik et al, ${ }^{5}$ a large proportion of deaths, especially in older patients, were attributed to causes other than breast cancer. Second, the difference may be due to coding errors. In identifying BCS mortality, we relied on death certificate records, which may overestimate intercurrent deaths with corresponding net underestimation of breast carcinoma deaths. ${ }^{17}$ Finally, it is possible that the negative impact of diabetes on all-cause mortality is partially caused by the increased toxicities of breast cancer therapy in diabetics.

Why did diabetic patients who received chemotherapy have higher BCS mortality than nondiabetic patients who received chemotherapy, whereas diabetic and nondiabetic patients who did not receive chemotherapy had similar BCS mortality? One possibility is that diabetics received less aggressive chemotherapy than nondiabetic patients. Our data showed that diabetics were, in fact, less likely to receive anthracyclines and taxanes, but these factors were accounted for in the multivariable analysis. We hypothesize that diabetic patients could be undertreated in other ways than drug choice, such as receipt of lower doses or shorter courses of chemotherapy or early discontinuation of chemotherapy because of toxicities. Another potential explanation is that diabetic patients could have lower compliance with hormonal therapies, either because of other medications or because of toxicities. Unfortunately, we were not able to evaluate these factors because of limitations of the Medicare data, but these issues could be addressed in future research studies on other data sets.

One of the strengths of this study is that it involves a large, population-based cohort of older patients with breast cancer. This study also includes patients who may be excluded from randomized trials. A major limitation of this observational study is selection bias. For instance, diabetic patients who are treated with chemotherapy are likely healthier than their counterparts who are not treated with chemotherapy. Therefore, the rates of toxicity in diabetic patients likely will be underestimated in this study. Although our analyses included the measurable confounding variables, unmeasured selection biases likely persist. Another limitation of our study is that SEER-Medicare data does not allow for assessment of severity of diabetes and associated end organ damage (although comorbidity index likely accounts for at least some of it). Additionally, we defined diabetes by the presence of at least one diagnosis code for diabetes, which could have resulted in overdiagnosis of diabetes. However, the result of this potential misclassification would have been to make differences between diabetic and nondiabetic patients less apparent. We also may have underascertained chemotherapy toxicity, because we used hospitalization billing 
records to identify toxicity. However, by limiting this study to toxicities that resulted in hospitalization, we were able to detect differences in serious toxicities between diabetic and nondiabetic patients, even if more subtle toxicities could not be reliably ascertained with claims. A minor limitation was that this study was restricted to patients older than 66 years of age; the results, thus, may not be applicable to a population of younger and, in general, healthier patients. Additional studies are needed to prospectively confirm these findings and to assess toxicities and outcomes in younger women who have diabetes and breast cancer.

In summary, we have shown that patients who have breast cancer and diabetes are slightly less likely to receive adjuvant chemotherapy. Among patients who receive chemotherapy, patients with diabetes are at an increased risk of developing chemotherapy-related toxicities, despite receipt of less-toxic regimens. We also have demonstrated that diabetes is associated with a significantly higher risk of all-cause mortality in elderly patients who have breast cancer. Breast cancer patients with diabetes who receive adjuvant chemotherapy have a higher risk of BCS mortality than patients without diabetes who receive chemotherapy. Despite these findings, patients with diabetes should continue to be offered standard chemotherapy regimens, because this study can- not definitely answer which chemotherapy offers the best risk-benefit ratio for patients with diabetes. Future studies are necessary to define the optimal management of breast cancer patients who have diabetes.

\section{AUTHORS' DISCLOSURES OF POTENTIAL CONFLICTS} OF INTEREST

The author(s) indicated no potential conflicts of interest.

\section{AUTHOR CONTRIBUTIONS}

Conception and design: Tomasz P. Srokowski, Shenying Fang,

Sharon H. Giordano

Administrative support: Sharon H. Giordano

Collection and assembly of data: Tomasz P. Srokowski, Shenying Fang, Sharon H. Giordano

Data analysis and interpretation: Tomasz P. Srokowski, Shenying Fang, Gabriel N. Hortobagyi, Sharon H. Giordano

Manuscript writing: Tomasz P. Srokowski, Shenying Fang, Gabriel N. Hortobagyi, Sharon H. Giordano

Final approval of manuscript: Tomasz P. Srokowski, Shenying Fang, Gabriel N. Hortobagyi, Sharon H. Giordano

\section{REFERENCES}

1. Wolf I, Sadetzki S, Catane R, et al: Diabetes mellitus and breast cancer. Lancet Oncol 6:103-111, 2005

2. Engel J, Kerr J, Schlesinger-Raab A, et al: Quality of life following breast-conserving therapy or mastectomy: Results of a 5-year prospective study. Breast J 10:223-231, 2004

3. Witt A, Yavuz D, Walchetseder C, et al: Preoperative core needle biopsy as an independent risk factor for wound infection after breast surgery. Obstet Gynecol 101:745-750, 2003

4. Fleming ST, Rastogi A, Dmitrienko A, et al: A comprehensive prognostic index to predict survival based on multiple comorbidities: A focus on breast cancer. Med Care 37:601-614, 1999

5. Yancik R, Wesley MN, Ries LA, et al: Effect of age and comorbidity in postmenopausal breast cancer patients aged 55 years and older. JAMA 285: 885-892, 2001
6. Hellman S: Stopping metastases at their source. N Engl J Med 337:996-997, 1997

7. Potosky AL, Riley GF, Lubitz JD, et al: Potential for cancer related health services research using a linked Medicare-tumor registry database. Med Care 31:732-748, 1993

8. Hebert PL, Geiss LS, Tierney EF, et al: Identifying persons with diabetes using Medicare claims data. Am J Med Qual 14:270-277, 1999

9. Du XL, Osborne C, Goodwin JS: Populationbased assessment of hospitalizations for toxicity from chemotherapy in older women with breast cancer. J Clin Oncol 20:4636-4642, 2002

10. National Cancer Institute: SEER-MedicareCalculation of comorbidity weights. http://healthservices .cancer.gov/seermedicare/program/comorbidity.html

11. Charlson $M E$, Pompei $P$, Ales $K L$, et al: A new method of classifying prognostic comorbidity in longitudinal studies: Development and validation. J Chronic Dis 40:373-383, 1987

12. Romano PS, Roos LL, Jollis JG: Adapting a clinical comorbidity index for use with ICD-9-CM administrative data: Differing perspectives. J Clin
Epidemiol 46:1075-1079, 1993; discussion 10811090

13. Centers for Disease Control: Prevalence of Diabetes: Number and Percent of U.S. Population with Diagnosed Diabetes. http://www.cdc.gov/ diabetes/statistics/prevalence_national.htm

14. Guastamacchia E, Resta F, Mangia $A$, et al: Breast cancer: Biological characteristics in postmenopausal type 2 diabetic women-Identification of therapeutic targets. Curr Drug Targets Immune Endocr Metabol Disord 3:205-209, 2003

15. Meyerhardt JA, Catalano PJ, Haller DG, et al: Impact of diabetes mellitus on outcomes in patients with colon cancer. J Clin Oncol 21:433440, 2003

16. Satariano WA, Ragland DR: The effect of comorbidity on 3-year survival of women with primary breast cancer. Ann Intern Med 120:104-110, 1994

17. Rutqvist LE: Validity of certified causes of death in breast carcinoma patients. Acta Radiol Oncol 24:385-390, 1985

\section{Acknowledgment}

We thank the Applied Research Program, National Cancer Institute; the Office of Research, Development, and Information, Centers for Medicare and Medicaid Services; Information Management Services; and the Surveillance, Epidemiology, and End Results (SEER) Program for the creation of the SEER-Medicare database. 